\title{
trans-gauche Energy Difference in Molecular Chains of Vinylidene Fluoride/Trifluoroethylene Copolymers in the Paraelectric Phase
}

\author{
Akira OdAJIMA and Geoffrey R. MITCHELL* \\ Department of Applied Physics, Faculty of Engineering, \\ Hokkaido University, Sapporo 060, Japan \\ *J. J. Thomson Physical Laboratory, University of Reading, \\ Whiteknights, Reading, RG6 $2 A F, U . K$.
}

(Received March 30, 1984)

\begin{abstract}
KEY WORDS Vinylidene Fluoride and Trifluoroethylene Copolymer / Ferroelectric Polymer / Ferroelectric Phase Transition / Paraelectric State / Local Conformation / Rotational Isomeric State Approximation /
\end{abstract}

Recently, the random copolymer of vinylidene fluoride/trifluoroethylene (P.VDF/ TrFE) has attracted much interest because it is the first ferroelectric polymer to exhibit a ferroelectric to paraelectric phase transition. The transition properties have been investigated by various methods such as $\mathrm{X}$-ray diffraction, ${ }^{1,2}$ infrared and Raman spectroscopies, ${ }^{2}$ dielectric relaxation, ${ }^{3,4}$ and nuclear magnetic resonance. ${ }^{5}$ The VDF-rich copolymers show a noticeable thermal hysteresis in which the Curie temperature, $T_{\mathrm{c}}$ upon heating is much higher than that upon cooling, but the TrFE-rich copolymers show less thermal hysteresis and a rather diffuse ferroelectric transition.

In this regard, Tashiro et $a .^{2 \mathrm{~b}}$ reported a composition dependence on energy difference between trans- and gauche-bondings in the paraelectric phase of $\mathrm{P} \cdot \mathrm{VDF}_{x} / \mathrm{TrFE}_{100-x}$. One of the authors $[\mathrm{AO}]^{6}$ has developed a statistical theory of phase transitions in polymer ferroelectrics based on Tashiro et al.'s findings and showed that the thermal hysteresis of $\mathrm{P} \cdot \mathrm{VDF}_{x} / \mathrm{TrFE}_{100-x}$ is associated with the local conformation of the molecular chains, and depends upon their composition ratio $x$ $(\mathrm{mol} \%)$. Tashiro et al.'s results are summarized as follows:

i) The IR spectra were measured for various compositions over a wide temperature range below the melting temperature, in which the bands $850 \mathrm{~cm}^{-1}$ and $610 \mathrm{~cm}^{-1}$ were attributed to the TT and TG conformers, respectively. The IR-absorbance ratio, $D_{840} / D_{612}$ was considered to be equivalent to the population ratio of the $\mathrm{TT}$ to $\mathrm{TG}$ pair, $\eta_{\mathrm{TT}} / \eta_{\mathrm{TG}}$, where $\eta_{\text {TG }}$ denotes the sum of populations of all TG pairs, $\eta_{\mathrm{TG}}+\eta_{\mathrm{T} \overline{\mathrm{G}}}+\eta_{\mathrm{GT}}+\eta_{\overline{\mathrm{GT}} \mathrm{T}}$.

ii) When $\ln \left(D_{840} / D_{612}\right)$ was plotted against the reciprocal temperature $T^{-1}$ in the paraelectric phase, the data gave a straight line. It was thus assumed that a trans-gauche energy difference, $\Delta u=U_{\mathrm{T}}-U_{\mathrm{G}}$, in the molecular chain may possibly be obtained from an Arrhenius-type temperature dependence:

$$
\ln \left(D_{840} / D_{612}\right)=-\Delta u / R T
$$

iii) The energy differences were estimated for various compositions, and $\Delta u$ changed its sign from negative to positive as the VDF $\mathrm{mol} \%$ increased. 
On the other hand, Tashiro et al.'s X-ray and IR measurements ${ }^{2}$ showed that the chains in the paraelectric phase consist of random sequences of $T_{3} G, T_{3} \bar{G}, T G$, and $T \bar{G}$ groups. Furthermore, a recent NMR study ${ }^{5}$ showed that such conformational defects move rapidly along the chain without interchain interactions and accompany the conformational change from trans to gauche. Since the interchain interactions are particularly weak compared to the interchain interactions in the paraelectric phase, the local conformation of the chains at temperatures above $T_{\mathrm{c}}$ may be determined by the energy difference between the trans and gauche bondings. However, Tashiro et al.'s estimation $^{2 b}$ is applicable only to random chains with independent rotation states.

Configurational statistics of an isolated chain with interdependent rotational potentials have been developed by the rotational isomeric state approximation. ${ }^{7}$ According to this theory, the ratio of the average number between TT pairs and TG pairs in polymethylene-like chains is expressed as

$$
\begin{aligned}
& \eta_{\mathrm{TT}} / \eta_{\mathrm{TG}} \\
& =\left[1-\sigma(\psi+\omega)+\sqrt{\{1-\sigma(\psi+\omega)\}^{2}+8} \sigma\right] / 8 \sigma
\end{aligned}
$$

and

$$
\sigma=\exp (\Delta u / R T)=\exp \left\{\left(E_{\mathrm{T}}-E_{\mathrm{G}}\right) / R T\right\}
$$

where $\sigma, \sigma \psi$ and $\sigma \omega$ denote the statistical weights associated with TG (TĞ) pairs, GG $(\bar{G} \bar{G})$ pairs and $G \bar{G}(\overline{G G})$ pairs, respectively. Geometrical parameters in $\mathrm{P} \cdot \mathrm{VDF}_{x} / \mathrm{TrFE}_{100-x}$ chains are similar to those in polymethylene chains. The somewhat greater length of the $\mathrm{C}-\mathrm{F}$ bond compared to $\mathrm{C}-\mathrm{H}$ and the greater van der Waals radius of fluorine, $1.35 \AA v s$. $1.20 \AA$ for hydrogen are the principal differences. Therefore, it is assumed that eq 2 is applicable to the case of $\mathrm{P} \cdot \mathrm{VDF}_{x} / \operatorname{TrFE}_{100-x}$.

If $\psi=\omega=1$ in eq 2 ,

$$
\eta_{\mathrm{TT}} / \eta_{\mathrm{TG}}=1 / 4 \sigma=\exp (-\Delta u / R T) / 4
$$

\begin{tabular}{|c|c|c|c|c|c|}
\hline & \multirow[b]{2}{*}{$(\mathrm{kcal} / \mathrm{mol})$} & \multicolumn{4}{|c|}{ VDF $\mathrm{mol} \%$} \\
\hline & & 37 & 55 & 65 & 72 \\
\hline \multirow{3}{*}{$\Delta u$} & $\psi=0\{\psi=1$ & $-0.5_{5}$ & -1.5 & $\sim 0$ & 2.0 \\
\hline & $\omega=0\{\psi=0$ & $-0.5_{5}$ & -1.5 & $\sim 0$ & 1.1 \\
\hline & $\omega=1 \quad \psi=1$ & -0.56 & -1.52 & $\sim 0$ & 0.69 \\
\hline
\end{tabular}

Table I. T-G energy difference $\left(\Delta u=E_{\mathrm{T}}-E_{\mathrm{G}}\right)$ of $\mathrm{P} \cdot \mathrm{VDF}_{x} / \mathrm{TrFE}_{100-x}$ in the paraelectric phase

The latter is the case of the random chain considered by Tashiro et al., ${ }^{2 \mathrm{~b}}$ since the bond rotational potentials are independent. Tashiro et al. obtained the values of $\Delta u$ for various VDF content from the $D_{840} / D_{612} v s . T^{-1}$ curve in the paraelectric phase, using eq 1 . Their results are listed on the bottom line in Table I. If $\psi=1, \omega=0$, the $G \bar{G}$ ( $\overline{G G}$ ) pairs are prohibited, but the GG $(\bar{G} \bar{G})$ pairs are free so that the molecular chain can adopt a local helical conformation. The values of $\Delta u$ for this case are listed in the middle line in Table I. The top line indicates the values of $\Delta u$ for various VDF content in the case of $\psi=\omega=0$. When $\Delta u \leqq 0$, there are little differences among the three cases, but when $\Delta u>0$, considerable differences appear.

The chains of $\mathrm{P} \cdot \mathrm{VDF}_{x} / \mathrm{TrFE}_{100-x}$ in the paraelectric state are packed in the hexagonal lattice with random sequences of $T_{3} G, T_{3} \bar{G}$, TG and $T \bar{G}$ in local, as mentioned above. Therefore, the top values are considered the most applicable for the trans-gauche energy difference of $\mathrm{P} \cdot \mathrm{VDF}_{x} / \mathrm{TrFE}_{100-x}$ in the paraelectric phase. When $\Delta u$ is positive in the paraelectric phase, the gauche form is more stable at lower temperatures. Thus, large configurational changes are required for the ferroelectric phase transition from such a paraelectric state, resulting in a first order transition with a wide thermal hysteresis. This is the case of $\mathrm{P} \cdot \mathrm{VDF}_{72} \mathrm{TrFE}_{28}$. The properties of its phase transition will be quantitatively described in terms of a statistical theory based on the present finding of the 
trans-gauche energy difference elsewhere. ${ }^{8}$

Acknowledgement. The authors wish to thank Dr. K. Tashiro of Osaka University for providing the IR-absorbance ratio data of $\mathrm{P} \cdot \mathrm{VDF}_{x} / \operatorname{TrFE}_{100-x}$ used in this study.

\section{REFERENCES}

1. a) A. J. Lovinger, G. T. Davis, T. Furukawa, and M. G. Broadhurst, Macromolecules, 15, 323 (1982); b) Idem., Polymer, 24, 1225, 1233 (1983).
2. a) K. Tashiro, K. Takana, M. Kobayashi, Y. Chatani, and H. Tadokoro, Polymer, 25, 195 (1984); b) Idem, Ferroelectrics, in press.

3. T. Furukawa, M. Ohuchi, A. Chiba, and M. Date, Macromolecules, in press.

4. N. Koizumi, N. Haikawa, and H. Habuka, Ferroelectrics, in press.

5. F. Ishii, A. Odajima, and H. Ohigashi, Polym. J., 15, 875 (1983).

6. A. Odajima, Ferroelectrics, in press.

7. P. J. Flory, "Statistical Mechanics of Chain Molecules," Interscience, New York, N. Y., 1969, Chapter 3.

8. A. Odajima and K. Yuasa, in preparation. 